\title{
Studies on the gastric mucosal microcirculation. 2 . Helicobacter pylori water soluble extracts induce platelet aggregation in the gastric mucosal microcirculation in vivo
}

\author{
N Kalia, S Jacob, N J Brown, M W R Reed, D Morton, K D Bardhan
}

\begin{abstract}
Background-The exact mechanisms by which Helicobacter pylori infection results in gastric mucosal injury are unclear.

Aims-To assess in vivo whether $\boldsymbol{H}$ pylori extracts could initiate an inflammatory response in the rat gastric mucosal microcirculation.

Methods-Extracts of $H$ pylori, Escherichia coli, or distilled water were administered topically to the gastric mucosa of anaesthetised animals. Fluorescence in vivo microscopy assessed macromolecular leakage of labelled albumin from mucosal vessels, leucocyte adherence/rolling, and platelet activity for 90 minutes.

Results-H pylori induced increases $(\mathbf{p}<0.001)$ in adherent platelet thrombi and circulating platelet emboli after five and 15 minutes respectively. Adherent platelet thrombi (mean of four per field of view) remained significantly increased throughout the experiment, but circulating emboli (maximum of five at 30 minutes) decreased with time. Leucocyte adherence did not occur although early transient rolling was observed. An 11\% increase $(p<0.02)$ in albumin leakage occurred after five minutes only. The induction of platelet aggregation was only observed following $H$ pylori administration.

Conclusion-This in vivo study demonstrated the ability of $\boldsymbol{H}$ pylori extracts to promote platelet aggregation within gastric mucosal microvessels. Recruitment of leucocytes was not observed. The results suggest that the early events associated with $H$ pylor $i$ infection are platelet aggregation with perhaps subsequent leucocyte recruitment by activated platelets. (Gut 1997; 41: 748-752)
\end{abstract}

Keywords: gastric mucosal microcirculation; Helicobacter pylori; fluorescence in vivo microscopy; platelet aggregation; leucocytes

Correspondence to: Dr N Kalia, Department of Surgical and Anaesthetic Sciences, Royal Hallamshire Hospital, Glossop Road, Sheffield S10 2JF, UK.

Accepted for publication 16 July 1997
The pathophysiology of Helicobacter pylori associated gastritis and peptic ulceration, and in particular, the mechanism by which this non-invasive bacterium induces mucosal inflammation, is poorly understood. However, many in vitro studies have shown that the bacterial surface proteins, extracted into water by centrifugation and vortex methods, are chemotactic for neutrophils and monocytes. ${ }^{1-4}$ In vivo investigations on the rat mesentery have also shown that these aqueous extracts cause leucocyte adherence in, and emigration from the microcirculation. ${ }^{56}$ These observations suggest that $H$ pylori recruits inflammatory cells by actively releasing or passively shedding cellular components which, after absorption into the mucosa, serve as chemoattractants for such cells. $^{7}$

$H$ pylori infection is, however, commonly localised to the stomach and therefore the findings of leucocyte involvement within the mesentery may not be mirrored in the gastric mucosa. This study aimed to assess in vivo the response of the gastric mucosal microcirculation to extracted $H$ pylori surface proteins. Fluorescence in vivo microscopy was used to determine whether $H$ pylori could induce alterations in leucocyte rolling and adherence, platelet activity, labelled albumin leakage from gastric mucosal capillaries and post-capillary venules (PCVs), and also to assess changes in vessel diameter and blood flow. Alterations in these microcirculatory parameters are characteristic features of acute inflammation ${ }^{8}$ and have been altered during other models of gastric injury induced by administration of a variety of toxic agents including ethanol and non-steroidal anti-inflammatory drugs. ${ }^{9} 10$

\section{Materials and Methods}

PREPARATION OF H PYLORI WATER EXTRACTS

$H$ pylori isolates, cultured from gastric antral biopsy specimens taken from six patients with active duodenal ulcer, were harvested into distilled water producing a suspension containing $10^{9}-10^{10}$ organisms $/ \mathrm{ml}$. The cell suspension was sonicated five times on ice for five seconds with 30 seconds between each sonication and then centrifuged at $12000 \mathrm{rpm}$ for 15 minutes at $4^{\circ} \mathrm{C}$. The initial supernatant was further centrifuged at $18000 \mathrm{rpm}$ for 30 minutes and the pellet discarded. The final supernatant was stored in aliquots of $0.5 \mathrm{ml}$ at $-20^{\circ} \mathrm{C}$ until use. Pooling of the $H$ pylori extracts obtained from six different patients avoided strain variations that could lead to conflicting results. It is assumed therefore that the antigenic preparation used is representative of the different superficial and secreted components found in 
different $H$ pylori strains. Control extracts of Escherichia coli were also prepared using pooled extracts from six patients to produce a suspension of $10^{9}-10^{10}$ organisms $/ \mathrm{ml}$. The extract was produced in a manner similar to that used for H pylori.

ANIMALS

Experiments were carried out on fasted adult male Wistar rats $(100-150 \mathrm{~g})$ anaesthetised with a subcutaneous injection of diazepam ( 5 $\mathrm{mg} / \mathrm{ml}$ ) and Hypnorm (fentanyl citrate 0.315 $\mathrm{mg} / \mathrm{ml}$ and fluanisone $10 \mathrm{mg} / \mathrm{ml}$ ) in a $1: 1$ ratio at a volume of $0.1 \mathrm{ml} / 100 \mathrm{~g}$ body weight. All experimental procedures were carried out in accordance with Home Office approval.

SURGICAL PROCEDURE

Tracheotomy was performed and an oesophageal thermistor probe was inserted and connected to a thermometer (Fluke, Washington, USA). A cannula was placed in the left carotid artery and was connected to a pressure transducer and physiograph (Micro-Med, Louisville, USA) to monitor mean arterial blood pressure, heart rate, and also to provide access for administration of fluorochromes.

After laparotomy a $1.0 \mathrm{~cm}$ incision was made with an electric microcautery in the middle of the anterior wall of the exteriorised stomach parallel to the "limiting ridge" (separates the squamous cell lined forestomach from the more distal glandular stomach). It was on the glandular portion of the posterior mucosal surface of the stomach that the observations were made. Care was taken to avoid burning through blood vessels.

The animal was placed on its left side onto a heating pad on a special perspex stage to maintain body temperature around $35-37^{\circ} \mathrm{C}$. A glass microscope slide was mounted on perspex pegs and the stomach was gently extended onto it and held in place by a stay suture.

The incision made in the anterior wall was opened using microclamps to allow visualisation of the posterior mucosal surface. The stomach was kept warm by covering with Saran wrap, an impermeable membrane, that also prevented dehydration.

FLUORESCENCE IN VIVO MICROSCOPY

The animal, warming pad, and perspex board were transferred to the stage of a Leitz microscope equipped with a mercury arc lamp for epi-illumination fluorescence light microscopy. A filter cube interposed into the light path of the mercury arc lamp permitted green light (530-560 nm) and blue light (450-490 $\mathrm{nm}$ ) to be selected for epi-illumination. Images of the preparation were monitored using a cooled coupled device camera (CCD, Hitachi) displayed on a high resolution monitor (Sony PVM-1443) and recorded by video (Sony SLV-373-UB) for later off line analysis. All instruments were calibrated prior to each experiment.

Following the surgical procedure, 30 minutes were allowed for blood pressure, heart rate, and body temperature to stabilise. These parameters were then monitored every 15 minutes for the remainder of the experiment. After the stabilisation period either acridine red, which fluoresces under green light $(0.1 \mathrm{ml} / 100 \mathrm{~g}$ body weight) or fluorescein isothiocyanate conjugated to bovine serum albumin, which fluoresces under blue light (FITC-BSA, $0.2 \mathrm{ml} /$ $100 \mathrm{~g}$ body weight) was injected via the carotid cannula. Acridine red labels leucocytes and FITC-BSA allows blood flow to be visualised. Under circumstances resulting in increased microvascular permeability to macromolecules, FITC-BSA can be observed to leak from the vasculature appearing as a flare in the interstitium. The interstitial fluorescence intensity is proportional to the degree of FITC-BSA leakage from the vessels. Details on the methodology and validity of this in vivo technique have been described previously. ${ }^{11} 12$

Animals were randomly divided into groups of six, receiving either $H$ pylori, $E$ coli, or distilled water. Three areas of the gastric mucosa were preselected in each animal for observation. These areas included the mucosal capillaries and at least one PCV ensuring that both these vessel types could be assessed. Changes in mucosal blood flow, macromolecular leakage, and vessel diameter were assessed. (In acridine red preparations only two areas of the mucosa were observed due to poorer resolution with this fluorochrome compared with FITC-BSA.)

Measurements were recorded from all selected regions five minutes after administration of the fluorochromes. Mucus present on the exposed stomach was removed prior to a single and topical application of $0.5 \mathrm{ml}$ of either $H$ pylori, E coli, or distilled water, which remained in contact with the mucosa for the 90 minute duration of the experiment. The procedure used to visualise the posterior mucosal surface allowed for the test extract to be simply held within the exposed region by the outer cautered edges of the anterior wall. Posttreatment recordings were made after five minutes and then every 15 minutes for 90 minutes.

\section{DATA COLLECTION AND QUANTIFICATION}

\section{Leucocyte and platelet activity}

The total number of adherent and rolling leucocytes within capillaries and PCVs in each field of view was determined off line from the videotapes. A leucocyte was considered adherent to the vessel endothelium if it remained stationary for $>30$ seconds. Adherent and rolling leucocytes were expressed as the number per field of view observed during a one minute period of continuous observation. As with the macromolecular leakage experiments, recordings were made at five minutes post-treatment and then every 15 minutes for 90 minutes.

Acridine red labels platelets as well as leucocytes but platelets can only be observed once aggregation has occurred. Therefore the sum of circulating platelet aggregates and adherent thrombi observed during a one minute period could also be quantitated in the same areas.

\section{Macromolecular leakage}

In order to quantitate FITC-BSA leakage from gastric microcirculation, an area adjacent to 


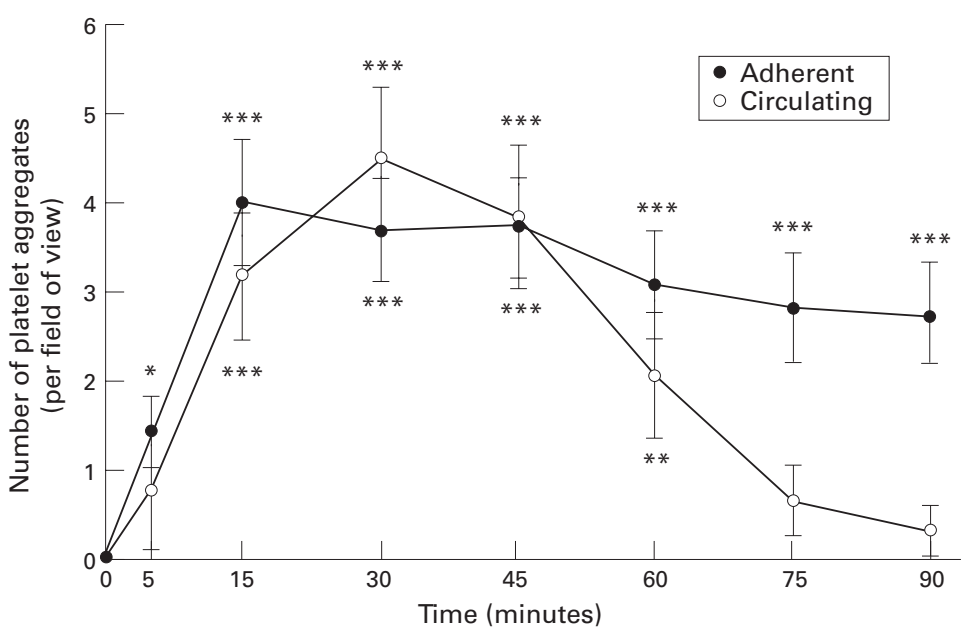
Figure 1: $\quad$ Number of adherent and circulating platelet aggregates in superficial gastric
mucosal vessels after exposure to $H$ pylori extracts. Results represent means (SEM); $n=6$. ${ }^{\star} p<0.05,{ }^{\star \star} p<0.01,{ }^{\star \star *} p<0.001$.

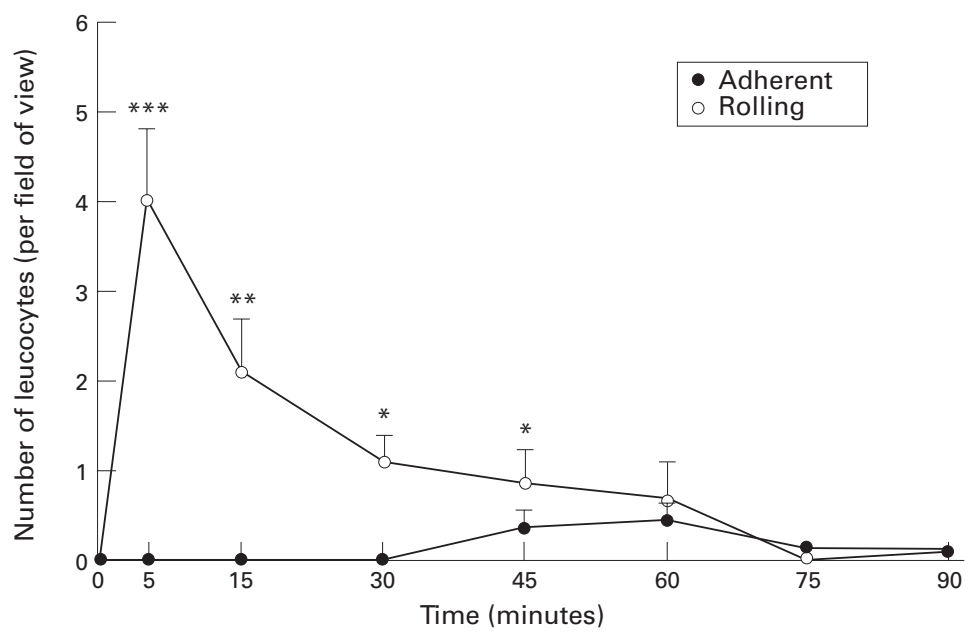

Figure 2: Number of adherent and rolling leucocytes in superficial gastric mucosal vessels after exposure to $H$ pylori extracts. Results represent means (SEM); $n=6 .{ }^{*} p<0.05$, ${ }_{\star \star}^{*} p<0.01,{ }^{\star *} p<0.001$.

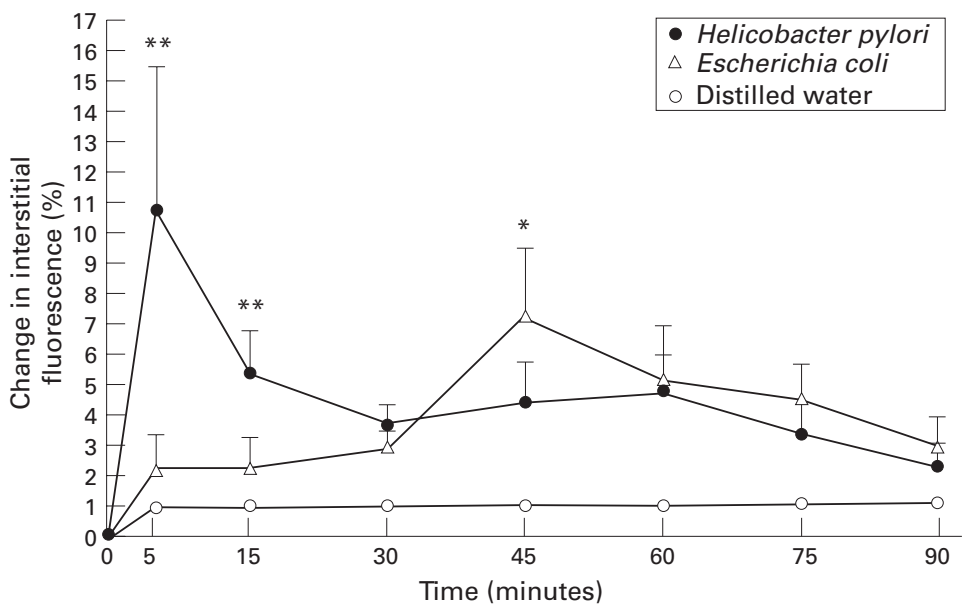

Figure 3: $\quad$ Effect of $H$ pylori extracts on macromolecular leakage of labelled albumin from superficial gastric mucosal post-capillary venules. Results represent means (SEM); $n=6$. ${ }^{\star \star} p<0.02,{ }^{\star} p<0.05$. three segments of the PCV and surrounding three separate capillaries was selected. Computerised image analysis (Image Pro Plus, Media Cybernetics, USA) was used to measure mean interstitial fluorescence at each time point during the experiment.

Vessel diameters and blood flow

Diameters of the PCVs and capillaries were also measured using computerised image analysis and mucosal vessel blood flow was assessed qualitatively as normal, reduced, or absent.

Statistical analysis

Results were tested for significance using the Mann-Whitney test for non-parametric data and the paired Student's $t$ test. Values of $\mathrm{p}<0.05$ were considered to be statistically significant. Data are presented throughout as means (SEM).

\section{Results}

$H$ pylori extracts induced platelet aggregation in gastric mucosal microvessels (fig 1). Adherent, but non-occlusive, platelet thrombi were only within the mucosal capillaries and not within the PCVs. A significant $(p<0.001)$ maximum effect occurred at 15 minutes when a mean of four aggregates was observed in the field of view. This was sustained for the duration of the experiment. Circulating platelet aggregates were observed within the PCVs and reached statistical significance after 15 minutes $(\mathrm{p}<0.001)$ but this effect was transient, returning to normal/control values at 75 minutes. Neither $E$ coli nor distilled water had significant effects on platelet activity.

Figure 2 shows the effects of $H$ pylori on leucocyte adherence and rolling. There was no significant increase in leucocyte adherence. However, the number of rolling leucocytes increased significantly $(\mathrm{p}<0.001)$ at five minutes but again this effect diminished after five minutes. Changes in leucocyte activity were not observed in control animals.

An $11 \%$ increase $(\mathrm{p}<0.02)$ in macromolecular leakage from PCVs was observed after five minutes incubation with the $H$ pylori extract. Values decreased rapidly thereafter approaching those seen with distilled water (fig 3). E coli showed a significant increase $(7 \% ; \mathrm{p}<0.05)$ in leakage at 40 minutes. Distilled water showed no significant change in macromolecular leakage at any time point. A similar pattern of events was observed in the mucosal capillaries.

None of the substances tested produced any significant change in the diameters of either the mucosal PCVs or capillaries which remained constant throughout the course of the experiment, although the technique does allow for changes to be detected (values for $H$ pylori: PCVs 26.3 (2.2) $\mu \mathrm{m}$ at five minutes and 25.4 (3.1) $\mu \mathrm{m}$ at 90 minutes; capillaries 12.9 (1.1) $\mu \mathrm{m}$ at five minutes and $12.7(0.99) \mu \mathrm{m}$ at 90 minutes). Although blood flow was assessed qualitatively, no visible changes appeared to be induced. The physiological parameters, heart rate, and blood pressure, remained constant throughout the course of the experiment for all study groups. 


\section{Discussion}

This in vivo study showed changes in the rat gastric mucosal microcirculation in response to $H$ pylori protein extracts. The most striking effect was the induction of significant numbers of circulating platelet emboli which was unique to $H$ pylori. Transient macromolecular leakage of fluorescently labelled albumin was also observed. However, leucocyte adherence to the endothelium, a frequently observed phenomenon in in vitro and histological studies, was not shown.

Significant increases in macromolecular leakage were observed immediately after administration of $H$ pylori. However, studies on the mesentery show a far greater effect on this parameter with increases as high as $70 \%$ after 30 minutes. $^{6}$ This could simply be due to mesenteric venules being more permeable to macromolecules than the gastric mucosal venules. ${ }^{6}$ It is also possible that the so-called "leak" in the stomach is an artefact brought about by the possible flare from the test substances on the mucosa. This seems unlikely, however, as the bacterial control, $E$ coli, induced "leak" at different time points, whereas distilled water, which was present on the mucosa continuously, did not. Kurose et al did however show a significant linear correlation $(r=0.891)$ between the number of emigrated leucocytes and the extent of albumin leakage in the mesentery. ${ }^{6}$ Subsequent monoclonal antibody studies to prevent leucocyte emigration showed that the early increase in leakage (at 10 minutes) was not dependent on leucocytes whereas the later increase (at 30 minutes) was leucocyte dependent. The lack of leucocyte activity may explain why no further increases in leakage were not observed. It is possible that the early leak observed in the current studies is due to some mechanism not involving leucocytes, perhaps mast cell mediated.

Significant increases in macromolecular leakage were also observed with $E$ coli. This bacteria's endotoxin has previously been observed to increase vascular permeability. ${ }^{13}$ Although it is documented that large amounts of $E$ coli endotoxin produce haemorrhagic mucosal lesions in the stomach, ${ }^{14}$ the significance of small and transient leakage, as observed in the current study for both $E$ coli and $H$ pylori, is unclear.

The formation of a mucus barrier subsequent to the topical administration of the $H$ pylori sonicates may explain the transient nature of both the macromolecular leakage and the platelet aggregation. Such a barrier would inhibit further proinflammatory substances within the $H$ pylori suspension diffusing across the mucosa to gain access into the interstitium. Additionally, adaptation of the mucosal microcirculation may occur after treatment, or the extract itself may lose its chemotactic potential with time.

Adherence of leucocytes was not a major feature of this study and this differs from previous in vivo studies performed on the rat mesentery. ${ }^{56}$ Although an immediate and significant increase in leucocyte rolling was observed, this was transient and is probably not biologically significant in the mechanisms of $H$ pylori induced damage in this particular model. Also, activation of leucocytes is usually manifested by adherence to the endothelial wall with subsequent extravasation into the interstitium. A longer period of superfusion with the $H$ pylori sonicate may be necessary to elicit such significant changes in leucocyte activity. The possibility exists that leucocytes may actually be present within the platelet aggregates, although the in vivo microscopy technique did not have the resolution for precise analysis. However, electron microscopy of the tissue (unpublished data) showed that the aggregates were purely platelet in nature. This differs from the observations made in vivo on the mesentery where the extract induced the formation of platelet-leucocyte aggregates.

Differences in the responses of these two tissue beds may be brought about by differences in the distribution of adhesion molecules on the endothelial cells of the mesentery and gastric mucosa. It seems reasonable to suggest that if leucocytes are an important component of the $H$ pylori induced disturbances, they would probably have shown an increased activity.

Most importantly, this study has shown directly a potential role for platelet aggregation in $H$ pylori induced gastric mucosal inflammation. It is now recognised that platelets themselves participate in the inflammatory response by acting as a potent source of inflammatory mediators and modulating the activity of other inflammatory cells. ${ }^{15}$ The early events in $H$ pylori induced inflammation may be the induction of platelet aggregation and the subsequent recruitment of leucocytes. In fact, an important role has been attributed to platelets in inflammatory bowel disease. ${ }^{16}$ It is quite possible that occlusion of microvessels by platelet thrombi may lead to focal mucosal infarction and therefore tissue necrosis.

N Kalia and this study were supported by Bardhan Research and Education Trust; registered charity number 328452.

1 Neilson H, Anderson LP. Chemotactic activity of Helicobacter pylori sonicate for human polymorphonuclear leucocytes and monocytes. Gut 1992;33:738-42.

2 Craig PM, Territo MC, Karnes WE, Walsh JH. Helicobacter pylori secretes a chemotactic factor for monocytes and neutrophils. Gut 1992;33:1020-3.

3 Mai UE, Perez-Perez GI, Wahl LM, Wahl SM, Blaser MJ, Smith PD. Soluble surface proteins from Helicobacter pylori activate monocytes/macrophages by lipopolysaccharide-independent mechanism. F Clin Invest 1991;87: 894-900.

4 Reymunde A, Deren J, Nachamkin I, Oppenheim D, Weinbaum G. Production of chemoattractant by Helicobacter pylori. Dig Dis Sci 1993;38:1697-1701.

5 Yoshida N, Granger DN, Evans DJ, Evans DG, Graham Yoshida N, Granger DN, Evans DJ, Evans DG, Graham
DY, Anderson DC, et al. Mechanisms involved in Helicobacter pylori-induced inflammation. Gastroenterology 1993;105:1431-40.

6 Kurose I, Granger DN, Evans DJ, Evans DG, Graham DY, Miyasuki M, et al. Helicobacter pylori-induced microvascular protein leakage in rats: role of neutrophils, mast cells and platelets. Gastroenterology 1994;107:70-9.

7 Mai UE, Perez-Perez GI, Allen JB, Wahl SM, Blaser MJ, Smith PD. Surface proteins from Helicobacter pylori exhibit chemotactic activity for human leucocytes and are present within the gastric mucosa. 7 Exp Med 1992;175: 517-25.

8 Kvietys PR, Granger DN. The vascular endothelium in gastrointestinal inflammation. In: Wallace J, ed. Immunopharmacology of the GI tract. Vol. 5. New York: Academic Press, macology of the

9 Kvietys PR, Twohig B, Danzell J, Specian RD. Ethanolinduced injury to the rat gastric mucosa-role of neutrophils and xanthine oxidase-derived free radicals. Gastroenterology 1990;98:909-20. 
10 Wallace JL, Keenan CM, Granger DN. Gastric ulceration induced by non-steroidal antiinflammatory drugs is a neutrophil dependent process. Am F Physiol 1990;259:G462-7.

11 Miller FN, Joshua IG, Anderson GL. Quantitation of vasodilator-induced macromolecular leakage by in vivo fluorescent microscopy. Microvasc Res 1982;24:56-67.

12 Kalia N, Jacob S, Brown NJ, Reed MWR, Bardhan KDB. Studies on the gastric mucosal microcirculation. 1. The nature of regional variations in response to ethanol injury.
Gut 1997;40:31-5.
Arroyo V, Rodes J. Endotoxin-induced ascites formation in the rat: partial mediation by platelet-activating factor. Hepatology 1989;10:788-94

14 Cheung LY, Reese RS, Moody FG. Direct effect of endotoxin on the gastric mucosal microcirculation and electrical gradient. Surgery 1976;79:564-8.

15 Braquet P, Touqui L, Shen TY, Vargaftig BB. Perspectives in platelet-activating factor research. Pharmacol Rev 1987;39. $97-145$

16 Collins CE, Rampton DS. Platelet dysfunction: a new dimension in inflammatory bowel disease. Gut 1995;36:5-8.

\section{Call for Patients with Familial Pancreatic Disease: The EUROPAC Register}

We are establishing a national UK register (EUROPAC) of families with hereditary pancreatitis, familial pancreatic cancer and where pancreatic cancer has occurred as part of a familial cancer syndrome. This collaboration in Liverpool is between the Department of Clinical Genetics (Dr Ian Ellis) and the Academic Department of Surgery (Professor John Neoptolemos). The data and samples are collected by behalf of ESPAC (the European Study Group for Pancreatic Cancer), Professor Markus Büchler, Berne, and Professor Hans Beger, Ulm. The study will collaborate with Dr David Whitcomb of the Midwest Multicenter Pancreatitis study group in the United States. We aim to recruit families who are prepared to donate blood for DNA studies. We hope to gain a clearer understanding of the genetic relationship between hereditary pancreatitis and familial pancreatic cancer, and develop screening protocols for individuals at risk.

Hereditary pancreatitis is associated with a mutation in the recently identified cationic trypsinogen gene. This mutation renders the enzyme active within the pancreas, leading to autodigestion. Individuals with recurrent pancreatitis have a greatly increased risk of developing pancreatic cancer, and there is some evidence that DNA analysis of cells from pancreatic fluid may be valuable in detecting premalignant changes which can predict the development of pancreatic adenocarcinoma.

The criteria for inclusion in the study are as follows:

- Hereditary pancreatitis: Three relatives with chronic pancreatitis in the absence of ethanol dependence, hypercalcaemia, or an obstructive cause.

- Familial pancreatic cancer: Two first degree relatives with pancreatic adenocarcinoma. Three or more relatives with pancreatic ductal adenocarcinoma. Pancreatic ductal adenocarcinoma in any two relatives where the sum of their ages is less than 110 years.

- Other familial cancer syndromes: A single documented pancreatic ductal adenocarcinoma in any family with an established familial cancer syndrome-for example, BRCA2, FAMMM, A-T, HNPCC, or FAP.

If you know of any suitable families who may be interested in joining the study, please contact: Fiona McRonald, Clinical Genetics, Alder Hey Children’s Hospital, Eaton Road, Liverpool L12 2AP. Tel: 01512525905.

Thank you for your help. 